\title{
La Ley de Igualdad entre Mujeres y Hombres garantiza, el Derecho de las Mujeres a la Propiedad Agraria en Ejidos y Comunidades en México
}

\section{The Law of Equality between Women and Men guarantees the Right of Women to Agrarian Property in Ejidos and Communities in Mexico.}

DOI: $10.46932 / \mathrm{sfjdv2n2-071}$

Received in: March 1st, 2021

Accepted in: May 30th, 2021

\author{
Marcial Reyes Cázarez \\ Doctorante en Género y Derecho por el Instituto Universitario Puebla. \\ Tecnológico Nacional de México campus Pátzcuaro. \\ Investigador Independiente del Estado de Michoacán. REIM_RECM81HMGE02 \\ Av. Tecnológico $\mathrm{N}^{\circ} 1$, Tzurumutaro, Pátzcuaro, Michoacán, México. \\ E-mail: mreyes@itspa.edu.mx ; marcial.rc@patzcuaro.tecnm.mx \\ Elvia Higuera Pérez \\ Maestra en Políticas Públicas y Gerencia Pública por la Universidad Nova Hispana. \\ Universidad Michoacana de San Nicolás de Hidalgo / Universidad Latina de América. \\ Fray Antonio de San Miguel N¹73, Centro Histórico de Morelia, Michoacán, México. \\ E-mail: elvidenise12@hotmail.com

\section{Daniel Reyes Cázarez} \\ Doctor en Ciencias de la Educación por la Universidad Contemporánea de las Américas. \\ Universidad Michoacana de San Nicolás de Hidalgo \\ Río Amatlán N 510, colonia Ventura Puente, Morelia, Michoacán, México \\ E-mail: despacho.danielrc@gmail.com

\section{Marcela Figueroa Ascencio} \\ Ingeniera en Desarrollo Comunitario por el Instituto Tecnológico Superior de Pátzcuaro \\ Corregidora $\mathrm{N}^{\circ} 178$, Tacámbaro, Michoacán, México. \\ E-mail: Marcefig555@gmail.com
}

\section{RESUMEN}

A través de la historia de México, la participación de la mujer en el ámbito agrario ha sido constante y efectivo, ya que las mujeres han luchado en igualdad de condiciones en diversos conflictos armados de tipo social, en las que se han recuperado grandes extensiones territoriales, siendo estas repartidas de forma igualitaria entre los jefes de familia, y la ley agraria descontextualizada de una realidad igualitaria y determinada para las condiciones de la década de los 50's, apertura el criterio de las comunidades y ejidos a regirse por ley de usos y costumbres, potenciando la exclusión y discriminación de forma más radical. Basándose en una Ley Agraria sexista donde el termino propietario, poseedor, hijos se considera desde un punto preferencial al varón en donde se nulifican los derechos de las mujeres, se toma en cuenta que la mujer únicamente puede acceder a la propiedad si es jefa de familia, pero ante los ojos de la ley Agraria y los usos y costumbres, solamente cuando esta enviuda, se considera posesionaria hasta que uno de sus hijos varones llega a la mayoría de edad y si esta no tuviera un hijo varón las condiciones de sus derechos se verían mermados ya que sería excluida del núcleo ejidal y/o comunal.

Derivado de esta realidad las Mujeres son excluidas de la tenencia de la tierra debido a la vulnerabilidad, al interior de los núcleos de posesión, la Ley Agraria, excluye y deja vulnerable a las mujeres debido a 
que el derecho a la propiedad no se ejerce. Con lo anterior y acorde a los acuerdos internacionales de los que el estado mexicano es parte, se propone, el empoderamiento de las mujeres violentadas de sus derechos humanos y a la tenencia de la tierra, ya que el derecho agrario en su autonomía derivado del artículo 27 de la Constitución Política de los Estados Unidos Mexicanos, emite que se deben crear leyes agrarias especiales que deroguen las normas comunes (Usos y costumbres), de las que podría depender el derecho agrario, así mismo es importante considerar que en la propuesta de políticas públicas se puede realizar la promoción y trabajo con la ciudadanía, tal como se especifica en la Ley para la Igualdad entre Hombres y Mujeres.

Palabras Clave: Igualdad, Derecho a la propiedad, Usos y costumbres.

\begin{abstract}
Throughout the history of Mexico, the participation of women in the agrarian sphere has been constant and effective, since women have fought on equal terms in various armed conflicts of a social nature, in which large territorial extensions have been recovered, The agrarian law was decontextualized from an egalitarian reality and determined for the conditions of the 50's, opening the criteria of the communities and ejidos to be governed by the law of uses and customs, promoting exclusion and discrimination in a more radical way.

Based on a sexist Agrarian Law where the term owner, possessor, children is considered from a preferential point to the male where the rights of women are nullified, it is taken into account that women can only have access to property if they are the head of the family, But in the eyes of the Agrarian Law and the uses and customs, only when she is widowed, she is considered a possessor until one of her sons reaches the age of majority, and if she does not have a son, the conditions of her rights would be diminished since she would be excluded from the ejidal and/or communal nucleus.

As a result of this reality, women are excluded from land tenure due to their vulnerability within the nuclei of possession, the Agrarian Law excludes and leaves women vulnerable because the right to property is not exercised. With the above and in accordance with the international agreements to which the Mexican State is a party, we propose the empowerment of women whose human and land tenure rights have been violated, since the agrarian law in its autonomy derived from Article 27 of the Political Constitution of the United Mexican States, It is also important to consider that in the proposal of public policies it is possible to promote and work with the citizenry, as specified in the Law for Equality between Men and Women.
\end{abstract}

Key words: Equality, Right to property, Usos y costumbres.

\title{
1 INTRODUCCIÓN
}

Los ejidos y comunidades agrarias, así como la pequeña propiedad, son las formas de tenencia de la tierra en la república mexicana, constituyendo y abarcando principalmente las áreas agrícolas productivas de importancia económica en el país.

La superficie constituida por ejidos y comunidades representan las áreas productivas de una gran diversidad en cuanto a producción agropecuaria se refiere, así mismo en el entorno de la vasta diversidad de recursos naturales tanto bióticos como abióticos de importancia y riqueza natural derivan, la diversidad 
de suelos y climas permiten la adaptabilidad de muchas especies y variedades agropecuarias. (Gamiz 2001)

La participación de la mujer en el contexto agrícola, ha sido fundamental para el desarrollo, cuidado y conservación de esta actividad y a su vez para la subsistencia de los seres humanos, ya que durante la evolución de la especie, la mujer ha sido proveedora de la alimentación de sus semejantes, siendo primordial el de origen vegetal, ya que de acuerdo a las actividades que desempeñaban en el periodo paleolítico se caracterizaban por ser recolectoras y existen datos en los que se le atribuyen créditos de ser las primeras trabajadoras de la tierra. (Vicent 1988)

Así mismo durante este periodo Paleolítico, cabe destacar que en las economías no existían excedentes, siendo este principio la mejor opción de igualdad social y de conservación de los recursos, en donde cada miembro del grupo era capaz de hacer lo que fuera necesario para sobrevivir, no importando la diferencia ni rol de género, simplemente determinando actividades por rango de edad, ya que no se especializaba el trabajo solo en algunos casos se debía de tener ciertas habilidades para ser chamanes y artesanos. (Martínez 2000)

Posteriormente conforme al desarrollo evolutivo de las diversas civilizaciones en México fueron modificadas algunas características propias del trabajo, el sustento social y la apropiación de los territorios colectivos.

La cultura Mixteca se refería en su propia lengua al territorio como Nuudzahui que significa pueblo de la lluvia. Nuu: es la tierra, lugar o pueblo. Dzahui: es lluvia. La historia de la cultura mixteca tiene raíces originadas en Yanhuitlan Oaxaca, hace aproximadamente 9000 años a.C. en esta época sus habitantes y se dedicaban a la cacería y la recolección.

Posteriormente entre los años 9000 a.C. - 1500 a.C., en la etapa de los nómadas, transcurrieron miles de años para que estos crearan comunidades aldeanas en donde comenzó la domesticación de especies con una agricultura experimental.

En los años 1500 a.C. - 600 a. C., progresivamente las aldeas fueron creciendo y floreciendo grandes ciudades denominándosele a esta la etapa de los centros urbanos. Así mismo del periodo 600 a. C. -800 d.C., los pobladores fueron abandonando estos centros y se fueron fundando los señoríos o cacicazgos. ${ }^{1}$

A través de la historia, la participación de la mujer en el ámbito agrario ha sido constante y efectivo, ya que las mujeres han luchado en igualdad de condiciones en diversos conflictos armados de tipo social en las que se han ganado grandes extensiones territoriales siendo estas repartidas de forma

\footnotetext{
${ }^{1}$ Cuadernillo informativo del templo y exconvento de Santo Domingo Yanhuitlan Oaxaca. Comisión de Vigilancia y Gobierno Municipal de Yanhuitlan. Pag. 1 parrafos 3 al 6.
} 
igualitaria entre los jefes de familia siendo estos una mayoría arrasadora, ya que la ley agraria así lo considera para su interpretación y cuando no se interpreta de esa forma, apertura el criterio de las comunidades y ejidos a regirse por ley de usos y costumbres potenciando la exclusión y discriminación de forma más radical. Basándose en una Ley agraria sexista donde el termino Propietario, poseedor, hijos se considera desde un punto de de vista dirigido al varón en donde se nulifican los derechos de las mujeres.(Riva Palacio 1972)

Tomando en cuenta que la mujer únicamente puede acceder a la propiedad si es jefa de familia, pudiéramos considerar que la mujer es jefa de familia ante los ojos de la ley Agraria y los usos y costumbres, solamente cuando esta enviuda y se le considera posesionaria hasta que uno de sus hijos varones llega a la mayoría de edad y si esta no tuviera un hijo varón las condiciones de sus derechos se verían mermados ya que sería excluida del núcleo ejidal y/o comunal y su propiedad pasa a convertirse en tierras de uso común.

En controversia existe la evolución jurídica respecto al derecho de las mujeres a la propiedad ejidal o comunal justificando que este es un derecho constitucional emanado en el artículo 27 de la promulgación de 1917, en donde se presume se considera el derecho a la propiedad por mujeres que se encuentren solteras o viudas con familia a su cargo, observando que en la actualidad las principales decisiones, en cuanto al futuro del patrimonio de los núcleos ejidales o comunales, se encuentra en el seno de los comisariados ejidales en el caso de los ejidos o bien en la representación de bienes comunales respecto a las comunidades indígenas. (Rivera 2003)

En este contexto las mujeres son excluidas de la tenencia de la tierra debido a prácticas de discriminación generadas desde la conquista de nuestro país en donde algunos indígenas decidieron no heredar las tierras o propiedades a sus hijas mujeres debido a que si se casaban con un español las tierras pasarían a ser propiedad de este, siendo las luchas armadas sociales de independencia y de la revolución mexicana tanto hombres como mujeres han peleado por el derecho de las mismas en igualdad de condiciones.

En este caso la ley agraria, excluye y deja vulnerable a las mujeres debido a que el derecho a la propiedad no se ejerce siendo la mujer únicamente resguardarte de la tenencia de la tierra, hasta el momento que su hijo varón cumpla la mayoría de edad esto en caso de que exista esta posibilidad y si no cuenta con hijos varones, la tenencia de la tierra queda acorde a los usos y costumbres del ejido o comunidad, en donde casi siempre son despojadas de la propiedad. (Tovar 2005)

Es importante argumentar que las mujeres son violentadas de sus derechos humanos y constitucionales con frecuencia y repetidamente ya que el derecho agrario en su autonomía derivado del 
artículo 27 constitucional emite que se deben crear leyes agrarias especiales que deroguen las normas comunes (Usos y costumbres) de las que podría depender el derecho agrario.

En el presente trabajo que se ha desarrollado durante la última década en la República Mexicana, se prioriza la importancia de visibilizar este problema ya que el derecho a la propiedad de las mujeres indígenas en el ámbito rural primordialmente depende de las decisiones de los hombres en los núcleos ejidales y tiene como objetivo; Identificar los diversos contextos de usos y costumbres que intervienen en el derecho a la propiedad de las mujeres en los núcleos ejidales y comunales en México para garantizar este derecho humano en apego a la protección constitucional del estado Mexicano y los compromisos internacionales en la materia de los que forma parte.

\section{DESARROLLO}

Un conocimiento fundamental en la evolución de la historia de la humanidad ha sido la actividad agrícola, desde la recolección, en apego al cuidado manejo y aprovechamiento de los recursos naturales como medio de subsistencia para su alimentación, cobijo y refugio.

Como parte esencial en el desarrollo de la humanidad ha sido la convivencia con otros seres, tales como relaciones entre seres vivos, en donde se comenzaba analizar ciertos comportamientos de los otros humanos y de animales y plantas a las cuales fueron identificando y experimentando propiedades y usos que proporcionaban en las labores diarias, y en el caso de la fauna observaban su comportamiento y los desplazamientos territoriales que estos realizaban para poder dominarlos y alimentarse de ellos posteriormente. (Vicent 1988)

Conforme pasó el tiempo y fueron generando habilidades y desarrollando sus capacidades de observación, dejaron de ser nómadas, al lograr reproducir técnicas especificas de plantas en las que solo recolectaban, pero ahora estableciéndolas en condiciones similares a donde se encontraban naturalmente y en el caso de algunas especies animales domesticándolas y convirtiendo granjas que a su vez preveían de alimento y vestido. Siendo lugares geográficos específicos cercanos a grandes ríos, lagos o lagunas para disponer del recurso hídrico para el desarrollo de sus grupos, que posteriormente se convirtieran en grandes civilizaciones. (Weigand 1997)

Estas civilizaciones comenzaron a determinar grandes extensiones territoriales para su establecimiento y desarrollo, debido a que cada vez se generaba una mayor población. Recalcando que aquellas civilizaciones que por su nivel de desarrollo, organización y productividad, generaban mayor cantidad de recursos y cabe destacar que se presume que la mujer fue la primera trabajadora de la tierra en términos de agricultura. 
A partir del establecimiento de estas civilizaciones en el planeta tierra, comienza la disputa por los territorios, en donde aquellos que desde estas épocas, hasta nuestra actualidad tienen mayor valor e interés adquisitivo aquellas superficies en donde son más abundantes los recursos naturales tales como los bosques como fabricas de conservación del agua y aquellos territorios que se ven favorecidos con la formación natural de vasos de agua mejor conocidos como lagos o lagunas en donde el agua es constante pero se encuentra en movimiento de un lugar geográfico a otro.

Siendo estos privilegios geográficos y la productividad de cada civilización un factor de constantes conflictos entre estas civilizaciones, por estos tres elementos; el recurso agua, la madera y frutos que producen los bosques, pero el principal botín de una confrontación o guerra eran las grandes extensiones territoriales que se ganaban dominando a la civilización derrotada o conquistada.

En México cuando comienzan estas civilizaciones, el pueblo azteca es uno de los referentes en materia de derechos agrarios ya que se respetaban estos derechos de forma igualitaria sin distinción de sexo, pero no se respetaba la clase a la que pertenecían, ya que si una mujer tenía mayor clase o abolengo tenia mayor derecho a la tenencia de la tierra. (Arias 1821)

La conquista de los españoles vino a quebrantar los derechos agrarios de los que gozaban las mujeres en el calpulli, siendo despojadas de los mismos, lo que significo una doble explotación de las mujeres en todo el periodo colonial. Ya que los padres no les heredaban las tierras a sus hijas indígenas por que los españoles las tomaban como botín de guerra, las despojaban de sus tierras y abusaban sexualmente de ellas para procrear su propia descendencia, quienes posteriormente serían los dueños y poseedores de esas tierras. (De la Torre 2004)

En el movimiento Armado de la Guerra de Independencia de 1810, tanto hombres como mujeres vuelven a luchar sin distinción de género para recuperar esas tierras de las que fueron despojados de la colonia, el que no tuvo éxito y por el contrario agudizo este problema, por lo que se genero el establecimiento de las compañías deslindadoras quienes se encargaron de un sinfín de irregularidades, que trajo consigo un despojo indiscriminado de tierras a las comunidades indígenas. (Arango 2005)

El problema Agrario fue empeorando hasta que se desato un nuevo movimiento armado en 1910, la Revolución Mexicana, nuevamente la historia se repite y hombres y mujeres en igualdad de condiciones se dispusieron a luchar por su derecho a una superficie de tierra donde poder cultivar. Como una esperanza de esta lucha armada surge en 1917 la promulgación de la constitución y la promesa de que en esta se incluirían el reconocimiento de la dotación y restitución de los derechos parcelarios propiedad de las comunidades indígenas. (Bocanegra 1982)

No todo fue favorable en cuanto quienes lucharon ya que esta constitución en el articulo 27 las leyes que esta deriva, limita los derechos de la mujer no fueron atendidos y dentro de los usos y costumbres 
de los ejidos y las comunidades tampoco fueron validos en cuanto a tres principios fundamentales, la cesión, donación y herencia de derechos parcelarios, a pesar de que el derecho agrario de la mujer se seguía ejerciendo al interior de las comunidades indígenas.

Los códigos agrarios de 1934 y 1942, excluyen nuevamente los derechos agrarios de las mujeres, bajo la figura de jefes de familia la personalidad de aquellos que tuvieran derechos para la tenencia de la tierra. En 1971 la Ley federal de la reforma agraria le da la facultad de ser retenedora o depositaria de los derechos, hasta que el primer hijo varón tuviera la mayoría de edad. (De la Torre 2004)

La Ley Agraria que reglamenta el artículo 27 constitucional en 1992, arrebata la posibilidad legal de acceder al derecho de la tierra por la única manera legal, aunque se da personalidad a la mujer, pero al igual se incluyen ejidos y comunidades al mercado de la oferta y la demanda, existiendo tratos turbulentos y desproveyendo de derechos a los sectores más vulnerables y marginados que principalmente hasta este momento eran la mayora de dueños y poseedores de las tierras nacionales.

Como parte fundamental de este trabajo considero esto antecedentes, en donde los roles de género en el tiempo de recolección y construcción durante el asentamiento de las poblaciones nómadas no se tenían definidos ni estereotipados mucho menos sexuados, hasta que comienzan las grandes batallas por la lucha de poderes y extensiones territoriales.

Pareciera ser que hemos olvidado este referente de nuestros antepasados ni las luchas históricas de hombres y mujeres en México en donde en pleno siglo veintiuno, realizamos acciones discriminatorias, tales como desconocimiento de una mujer sino representa ser jefa de familia, para heredar un título de propiedad o certificado parcelario, engaños o contratos turbulentos para despojar al indígena de su propiedad, desconocer la propiedad hereditaria de niños, ancianos o personas con capacidades diferentes por gente corrupta, deshonesta y oportunista llena de ambición, siendo estas acciones recurrentes a nivel internacional, nacional y local. (Díaz Gordillo 2008)

Es importante entonces revisar tal como lo presentamos a continuación que dentro de la carta magna de los Estados Unidos Mexicanos, cuyo documento se expresa como la Constitución Política de los Estados Unidos Mexicanos, la cual fue promulgada el lunes 5 de febrero de 1917, en el Diario Oficial por el Órgano del gobierno provisional de la república mexicana, en el tomo V, 4ta época, número 30. En donde el Artículo 27 constitucional establece:

\footnotetext{
"La propiedad de las tierras y aguas comprendidas dentro de los límites del territorio nacional, corresponde originariamente a la Nación, la cual ha tenido y tiene el derecho de transmitir el derecho de ellas a los particulares, constituyendo la propiedad privada".
}

En este párrafo de la constitución original la cual aun no ha sido reformada habla de la propiedad de las tierras y aguas de forma general en donde no se especifica, ni se da acceso o derecho a la propiedad 
ni a hombres ni mujeres siendo una disposición general y que en términos jurídicos se aplicaría bajo tal efecto.

Posteriormente en el párrafo tercero de la misma constitución, analizamos que los principios constitucionales determinan la relación entre la propiedad y la apropiación de los recursos disponibles en las tierras y aguas Nacionales la cual contempla los siguientes principios:

\begin{abstract}
"La Nación tendrá en todo tiempo el derecho de imponer a la propiedad privada las modalidades que dicte el interés público, así como el de regular el aprovechamiento de los recursos naturales susceptibles de apropiación, para hacer una distribución equitativa de la riqueza pública y para cuidar de su conservación. Con este objeto se dictaran las medidas necesarias para el fraccionamiento de los latifundios; para el desarrollo de la pequeña propiedad, para la creación de nuevos centros de población agrícola con las tierras y aguas que les sean indispensables; para el fomento de la agricultura y para evitar la destrucción de los elementos naturales y los daños que la propiedad pueda sufrir en perjuicio de la sociedad. Los pueblos rancherías y comunidades que carezcan de tierras y aguas, o no las tengan en cantidad suficiente para las necesidades de su población, tendrán derecho a que se les dote de ellas tomándolas de las propiedades inmediatas, respetando siempre la pequeña propiedad. Por tanto, se confirman las dotaciones de terrenos que se hayan hecho hasta ahora de conformidad con el Decreto de 6 de enero de 1915".
\end{abstract}

En el marco de este principio jurídico que establece el párrafo anterior se especifica que todo se apega a lo establecido en el Decreto de 6 de enero de 1915, el cual especifica:

\begin{abstract}
"CONSIDERANDO Que una de las causas más generales del malestar y descontento de las poblaciones agrícolas del país, ha sido el despojo de los terrenos, de propiedad comunal o de repartimiento, que les habías sido concedidos por el gobierno colonial como medio de asegurar la existencia de la clase indígena, y que, a pretexto de cumplir con la ley de 25 de junio de 1856 y demás disposiciones que ordenaron el fraccionamiento y reducción a propiedad privada de aquellas tierras entre los vecinos del pueblo a que pertenecían, quedaron en poder de unos cuantos especuladores; Que en el mismo caso se encuentran multitud de otros poblados de diferentes partes de la República, y que, llamados congregaciones o rancherías, tuvieron origen en alguna familia o familias que poseían en común extensiones más o menos grandes de terreno, los cuales siguieron conservándose indivisos por varias generaciones, o bien en cierto número de habitantes que se reunían en lugares propicios, para adquirir y disfrutar mancomunadamente, aguas, tierras y montes, siguiendo la antigua y general costumbre de los pueblos indígenas".
\end{abstract}

Como podemos apreciar en el Decreto anterior, se expresa textualmente en los últimos renglones, la limitante que la general costumbre de los pueblos indígenas para la toma de decisiones, respecto al derecho a la propiedad no es la más adecuada.

Respecto a la Constitución Política de los Estados Unidos Mexicanos, que actualmente rige a la Nación genera aun mayor incertidumbre respecto a la propiedad, no solo de las mujeres sino de todas las personas, en donde se limita el acceso a la propiedad, ya que su principio vigente el párrafo tercero ha sido modificado con tres diferentes reformas y dicta lo siguiente:

"La nación tendrá en todo tiempo el derecho de imponer a la propiedad privada las modalidades que dicte el interés público, así como el de regular, en beneficio social, el aprovechamiento de los elementos naturales susceptibles de apropiación, con objeto de hacer una distribución equitativa 
de la riqueza pública, cuidar de su conservación, lograr el desarrollo equilibrado del país y el mejoramiento de las condiciones de vida de la población rural y urbana. En consecuencia, se dictarán las medidas necesarias para ordenar los asentamientos humanos y establecer adecuadas provisiones, usos, reservas y destinos de tierras, aguas y bosques, a efecto de ejecutar obras públicas y de planear y regular la fundación, conservación, mejoramiento y crecimiento de los centros de población; para preservar y restaurar el equilibrio ecológico; para el fraccionamiento de los latifundios; para disponer, en los términos de la ley reglamentaria, la organización y explotación colectiva de los ejidos y comunidades; para el desarrollo de la pequeña propiedad rural; para el fomento de la agricultura, de la ganadería, de la silvicultura y de las demás actividades económicas en el medio rural, y para evitar la destrucción de los elementos naturales y los daños que la propiedad pueda sufrir en perjuicio de la sociedad. Párrafo reformado DOF 06-02-1976, 10 08-1987, 06-01-1992"

Respectivamente la Ley Agraria, siendo reglamentaria de lo establecido en la Constitución Política de los Estados Unidos Mexicanos establece lo siguiente:

"Artículo 1o.- La presente ley es reglamentaria del artículo 27 Constitucional en materia agraria y de observancia general en toda la República".

“TITULO TERCERO DE LOS EJIDOS Y COMUNIDADES Capítulo I De los Ejidos Sección Primera Disposiciones Generales Artículo 9o.- Los núcleos de población ejidales o ejidos tienen personalidad jurídica y patrimonio propio y son propietarios de las tierras que les han sido dotadas o de las que hubieren adquirido por cualquier otro título".

En este párrafo anterior de la Ley Reglamentaria, es importante visualizar que se expresa la personalidad jurídica a los núcleos de población y el derecho a la propiedad de sus extensiones territoriales adquiridas.

Artículo 10.- Los ejidos operan de acuerdo con su reglamento interno, sin más limitaciones en sus actividades que las que dispone la ley. Su reglamento se inscribirá en el Registro Agrario Nacional, y deberá contener las bases generales para la organización económica y social del ejido que se adopten libremente, los requisitos para admitir nuevos ejidatarios, las reglas para el aprovechamiento de las tierras de uso común, así como las demás disposiciones que conforme a esta ley deban ser incluídas en el reglamento y las demás que cada ejido considere pertinentes.

En este párrafo anterior se faculta a las autoridades ejidales a regirse bajo su reglamento interno, dejando a libre albedrio, la interpretación Constitucional, de los términos de la Ley Reglamentario y del mismo Reglamento de la Ley, propiciando un problema de discriminación y de limitación del derecho a la propiedad ejidal de la mujer.

Artículo 11.- La explotación colectiva de las tierras ejidales puede ser adoptada por un ejido cuando su asamblea así lo resuelva, en cuyo caso deberán establecerse previamente las disposiciones relativas a la forma de organizar el trabajo y la explotación de los recursos del ejido, así como los mecanismos para el reparto equitativo de los beneficios, la constitución de reservas de capital, de previsión social o de servicios y las que integren los fondos comunes".

Es importante establecer que en estos artículos expuestos anteriormente se faculta a la asamblea a la resolución de los acuerdos internos de la vida comunitaria y del desarrollo interno tanto del ejido 
“Artículo 12.- Son Ejidatarios los hombres y las mujeres titulares de los derechos ejidales".

Artículo que pende de un hilo muy delgado, hablando en términos de derecho a la propiedad ya que acorde a lo establecido en el reparto agrario en México, con base en los artículos de esta propia Ley que siguen vigentes establecen lo siguiente:

“Artículo 15.- Para poder adquirir la calidad de ejidatario se requiere: I. Ser mexicano mayor de edad o de cualquier edad si tiene familia a su cargo o se trate de heredero de ejidatario; y II. Ser avecindado del ejido correspondiente, excepto cuando se trate de un heredero, o cumplir con los requisitos que establezca cada ejido en su reglamento interno".

Esta Ley a pesar de la enmienda que se le realiza como anteriormente lo vimos en el artículo 12 donde se reconoce a la mujer como ejidataria, durante el transcurso del reparto agrario se entregaron los certificados parcelarios, únicamente a los jefes de familia, siendo muy pocas excepciones y acorde a las condiciones de cada entidad federativa, el entregar a las viudas de los hombres caídos durante la lucha armada de la Revolución Mexicana, siendo muy limitados estos certificados y bajo la restricción de que estas mujeres fueran posesionarias, hasta que un hijo varón cumpliera la mayoría de edad para que ejerciera dichos títulos de propiedad y se convirtiera en el jefe de familia y por ende en ejidatario.

Siendo que si por cualquier otra situación la mujer que tuviera títulos de propiedad y su condición de madre no le permitiera tener un hijo varón al momento de su fallecimiento esa fracción del ejido o comunidad pasaría a ser parte de las áreas comunes del núcleo ejidal o comunal. Esta situación prevalece en gran medida en el territorio nacional y las mujeres derivado a la baja preparación académica en zonas rurales, desconocen de los principios legales de derecho a la propiedad.

Así mismo es importante mencionar que la Ley Reglamentaria del artículo 27 Constitucional “Ley Agraria y su Reglamento", aun se encuentran bajo una redacción discriminatoria, ya que en todo momento se expresa dirigida al ejidatario, es decir sin perspectiva de género.

Lo expresado anteriormente, no ha garantizado lo establecido en la Ley para la Igualdad entre Mujeres y Hombres hasta la fecha siendo que esta fue en el Diario Oficial de la Federación el 2 de Agosto de 2006 y su última reforma fue publicada en el mismo Diario el 14 de Julio de 2018, la cual establece en el Capítulo Cuarto, Artículo 16, Fracción V lo siguiente:

Artículo 16.- De conformidad con lo dispuesto en la presente Ley y las leyes locales de la materia, corresponde a los Municipios:

V. Fomentar la participación social, política y ciudadana dirigida a lograr la igualdad entre mujeres y hombres, tanto en las áreas urbanas como en las rurales. 
Lo establecido en este Artículo 16, no ha cumplido el objetivo en la práctica toda vez que las condiciones presentes en cuanto a materia de derecho a la propiedad sigue en una misma tendencia y no existen Políticas Públicas encaminadas a un verdadero empoderamiento de la mujer, transgrediendo así mismo el Artículo 17, Fracción IV que establece lo siguiente:

Artículo 17.- La Política Nacional en Materia de Igualdad entre mujeres y hombres deberá establecer las acciones conducentes a lograr la igualdad sustantiva en el ámbito, económico, político, social y cultural.

IV. Promover la igualdad de acceso y el pleno disfrute de los derechos sociales para las mujeres y los hombres;

Es entonces en donde la percepción de la ciudadanía, forma parte de un doble discurso, por una parte el gobierno habla de equidad de género y del empoderamiento de las mujeres y por otro lado establece Techos de Cristal, donde supone que las mujeres tienen el derecho a la propiedad y por ende la toma de decisiones.

\section{CONCLUSIONES}

La propiedad social en el estado mexicano, la tenencia de la tierra es conformada por las propiedades privadas individuales llamadas pequeña propiedad, los ejidos y las comunidades agrarias, designándose a estas dos últimas como propiedad social o núcleos agrarios. Los ejidos y comunidades constituyen modalidades de propiedad de la tierra exclusivas del país y son producto de la reforma agraria (1934 y 1992).

La propiedad social abarca 198.5 millones de hectáreas; de ellas, las posesiones sociales cubren una extensión cercana a 102 millones de ha (los ejidos 84.5 millones y las comunidades 17.4 millones), equivalente a $53.4 \%$ de la superficie. A la propiedad privada (incluyendo las colonias agrícolas) pertenecen cerca de 79 millones de hectáreas, $39.8 \%$ de la extensión del suelo nacional, mientras que los terrenos nacionales ascienden a 7.7 millones de hectáreas (3.9\%). (PROCEDE 2004)

Los núcleos agrarios se conforman de 5653637 ejidatarios, comuneros y posesionarios: los dos primeros representan $74.5 \%$ y los posesionarios, $25.5 \%$ del total. La titularidad de los derechos parcelarios entre los ejidatarios expresa una fuerte masculinidad pues $80.2 \%$ de sus titulares son hombres y $19.8 \%$ mujeres. (INEGI 2007)

Ante estas cifras es importante mencionar que dentro de las autoridades y toma de decisiones en los núcleos agrarios la participación de las mujeres aun es más limitada ya que ni el 1\% de aquellas que tienen la fortuna de tener una titularidad, participan ni en el Comisariado Ejidal ni en la Representación de los Bienes Comunales. Un claro ejemplo de la Aplicación de la Cima y los Componentes de los Techos 
de Cristal, en donde los puestos de toma de decisiones están conformados por hombres, lo que propicia altos niveles de discriminación, violencia contra la mujer, actitudes machistas las cuales son invisibilizadas, toda vez que las asambleas son a puerta cerrada. (Mireya Martí Reyes, 2021)

Esta tendencia transgrede a los principios de la Convención Sobre la Eliminación de Todas las Formas de Discriminación Contra la Mujer CEDAW, de la cual el estado mexicano forma parte desde el 17 de julio de 1980 y entrada en vigor el 3 de septiembre de 1981. Cuyos artículos 1; Art. 2 incisos a, b, c, e y f; Art. 5 inciso a; Art. 7 incisos b y c; Art. 10; Art. 11 apartado 2 inciso c; Art 14 Apartados 1 y 2 incisos f, g, h; Art.15 Apartados 1, 2 y 4 y finalmente el Art. 16 Apartado 1 inciso h, los cuales están siendo violentados ya que se fundamenta en el presente trabajo que se transgreden en diversos aspectos los derechos de la mujer y se limita el derecho a la propiedad de las mismas.

También es importante describir que de acuerdo a las disposiciones de la Convención Interamericana para Prevenir, Sancionar y Erradicar la Violencia contra la Mujer o Convención de Belém do Pará, se ve trasgredida en los siguientes artículos: Art. 4 incisos f, g, j; Art. 5 inciso b, Art. 7 incisos c, d, f, g y h estos tres últimos de vital importancia de recalcar; Art. 8 incisos a, b, e, i; Artículos 11 y 12.

Finalmente es importante recalcar que la misógina distribución de la propiedad y la actual legislación vigente en la materia, limitan el acceso y derecho a la propiedad de las mujeres en México por un contexto de Usos y Costumbres, misóginos y descontextualizados que impiden el empoderamiento de la mujer en los núcleos agrarios y deja a la mujer distante de la toma de decisiones y la participación en representación de los núcleos agrarios, dejando en situación de vulnerabilidad a sus hijos e hijas, pareciendo que por el hecho de haber nacido mujeres no tienen el derecho a tener una propiedad de la cual puedan echar mano para la autoproducción de sus insumos para la autoalimentación. (Banco Mundial 2006) 


\section{BIBLIOGRAFIA}

Banco Mundial. (2006). Reposicionando la nutrición como prioridad para el desarrollo. Una estrategia para intervenciones de gran escala. Washington: Banco Mundial.

INEGI. (2007). Anuario estadístico. Michoacán de Ocampo. Instituto Nacional de Estadística, Geografía e Informática, Gobierno del Estado de Michoacán de Ocampo.

PROCEDE. (2004). Núcleos agrarios. Tabulados básicos por municipio. Michoacán de Ocampo. Programa de Certificación de Derechos Ejidales y Titulación de Solares, abril de 2002 a agosto de 2004.

SRA. (1998). La transformación agraria. Origen, evolución, retos, testimonios. Secretaría de la Reforma Agraria. México.

Arango, J.C. (2005). Desigualdad y exclusión en Colombia (1990-2000). Los problemas nutricionales desde una aproximación del enfoque de las capacidades humanas. Tesis de maestría obtenida no publicada. Universidad de Antioquia, Colombia.

Arias, J. D. (1821). “México independiente.” . En: México a través de los Siglos. México. Tomo VII. Editorial Cumbre. p. 158.

Avila, T. O. (2007). Caracterización de Tres Sistemas de Manejo de Recursos Naturales en un Ecosistema Templado de México. Resumen del 2do Congreso Brasileño de Agroecologia (págs. 241243). Rio de Janeiro: Revista Brasileña de Agroecologia.

Bocanegra, J. M. (1892). Memorias para la Historia de México Independiente. México: NEHRM, 1985, pp. 444-445.

De la Torre Villar, E. (2004). Religión, Poder y Autoridad en la Nueva españa. Serie Historia Novohispana $72,446$.

Delgado, A. S. (2011). Escuela de Formación Integral para la Promoción del Desarrollo Local Sustentable (primera ed.). Morelia, Michoacán, México: Morevalladolid, S. de R. L. de C.V

Díaz Gordillo, M. C. (2008). Una Ley Agraria para el futuro El Cotidiano [en línea], 23 (enero-febrero) : [Fecha de consulta: 11 de mayo de 2016] Disponible en: $\leq$ http://www.redalyc.org/articulo.oa?id=32514703> ISSN 0186-1840.

Gamiz, M. (2001) Constitución Política de los Estados Unidos Mexicanos comentada, 4ª Ed. Noriega Editores, México 2001.

Martí M., Cervera C. (2021). Mujeres Académicas frente al techo de cristal. Un estudio de caso en educación superior. South Florida Journal of Development, 2(2), 1853 - 1861.

Martínez A., Rentería C. (2000). El patrimonio arqueológico de México como recurso turístico: riesgos, ventajas y condiciones de explotación turística. En: Sitio del Museo Arqueológico Nacional (Madrid, España); búsqueda:

Congresos. http://www.man.es/archivos/congresos/arqueologiayturismo/textos/conferencias/martinez\%20muriel/ma rtinez\%20muriel_intro.htm. Consultado el 27 de diciembre de 2004. 
Riva Palacio, V. (1972) “La mal casada”. En: El Correo del Comercio, Segunda época. Núm. 486, 21.

Rivera, I. (2003). "Tenencia de la tierra y derechos agrarios"; en: Sitio oficial de SAGARPA (Secretaría de Agricultura, Ganadería, Desarrollo Rural, Pesca y Alimentación): http://ganaderia.sagarpa.gob.mx; en el vínculo: http://www.sagarpa.gob.mx/sdr/evets/sm_jovenes/pdfs/1_eirr_sra.pdf. Consultado el 28 de diciembre de 2004.

Tovar, J. O. (2005). Manual Práctico del Derecho Agrario (segunda ed.). Morelia, Michoacán , México: Secretaria de Difusión Cultural y Extensión Universitaria UMSNH.

Vicent García, J.M. (1988) El origen de la economía productora. Breve introducción a la historia de las ideas, en LOPEZ, P., El neolítico en España, p.11-58.

Weigand, P., Williams, E. (1997). "Adela Bretón y los inicios de la arqueología en el occidente de México", en Relaciones. Estudios de Historia y Sociedad, vol. XVIII, primavera, núm. 70, 1997, 127-256. 